\title{
FAILURE TO THRIVE
}

\section{INTRODUCTION}

Failure to thrive (FTT) is a descriptive term that refers to a child with relative undernutrition and subsequent inadequate growth over time, when compared to other children of similar age, gender, and ethnicity. Several definitions have been proposed based on abnormal anthropometric criteria, but none is uniformly accepted. The etiology of FTT is often multifactorial and results from a complex interplay between psychosocial, behavioral, and physiological factors. Ultimately, this interaction leads to one of three outcomes - inadequate caloric intake (in the setting of normal or excessive metabolic demands), inadequate absorption of calories, or impaired utilization of absorbed calories. FTT is often successfully managed in the outpatient setting. However, hospitalization may be necessary for very complex situations, when a child's safety is in question, or when outpatient management has not been successful. It is estimated that FTT accounts for 1 to $5 \%$ of all pediatric hospitalizations. Pediatric hospitalists should use evidence-based approaches to guide evaluation and management, provide leadership for multidisciplinary teams, and coordinate care to optimize outcomes.

\section{KNOWLEDGE}

Pediatric hospitalists should be able to:

- Describe the differential diagnosis of FTT for children of varying chronological and developmental ages recognizing that most children with FTT do not have an underlying medical disorder.

- Explain why infants and toddlers are at greater risk for FTT than older children.

- Describe the association between FTT and child abuse and neglect.

- Describe normal growth patterns for children and the sequential effect of undernutrition on weight velocity, height velocity, and head growth.

- Describe the key historical or physical examination findings that may indicate a psychosocial, behavioral, or physiological factor contributing to poor growth.

- Provide indications for admission to the hospital and state criteria for determining the appropriate level of care (ward vs. intensive care unit).

- Describe the goals of hospitalization including stabilization, diagnosis, treatment, observation, and education.

- Discuss the importance of observation of feeding behaviors and recording of nutritional intake over time in the evaluation of FTT.

- State the indications for laboratory, radiographic, or other testing in the evaluation of FTT.

- Discuss the indications for consultation with a pediatric speech or occupational therapist, nutritionist, gastroenterologist or other subspecialist.

- Discuss the need for catch-up calories in FTT, as well as the methods by which to achieve adequate caloric supplementation.

- Define the refeeding syndrome, and describe methods to prevent it or lead to its early detection.

- Discuss potential sequelae of FTT (such as behavioral or developmental abnormalities, increased susceptibility to infections, and others) and list the risk factors for worse outcomes.

- Summarize the literature on the impact of hospitalization on the evaluation, management, and outcomes for FTT.

\section{SKILLS}

Pediatric hospitalists should be able to:

- Stabilize patients presenting with metabolic abnormalities, cardiopulmonary compromise, or other urgent problems as a result of dehydration, malnutrition, or an abnormal pathophysiological state.

- Obtain a thorough patient history, including a detailed social, family, dietary and feeding history, attending to markers of abnormal behavioral or psychosocial factors.

- Perform a directed physical examination, including careful measurement of anthropometric data, attending to findings that may indicate an underlying medical condition or child abuse and neglect.

- Correctly utilize standardized growth charts to identify isolated growth abnormalities and to assess the growth pattern over time.

- Directly observe and correctly interpret a feeding session, with attention paid to feeding behavior and the child-caregiver interactions. 
- Critically assess the level of evidence and risk/benefit ratio for an expanded diagnostic evaluation.

- Interpret basic tests and identify abnormal findings that require further testing or consultation.

- Correctly calculate caloric needs and adjust feeding regimens to maximize weight gain while avoiding gastrointestinal compromise.

- Correctly identify the need for and efficiently access appropriate consultants and support services needed to provide comprehensive care.

- Coordinate care with subspecialists, the primary care provider and other services and arrange for an appropriate transition plan with detailed follow-up plans for after hospital discharge.

\section{ATTITUDES}

Pediatric hospitalists should be able to:

- Consider the concerns of the family/caregiver when obtaining a history, developing a diagnostic approach, and offering anticipatory guidance and management options.

- Provide education to the family/caregiver on FTT, with specific focus on patient-specific underlying diagnoses.

- Communicate effectively with the family/caregiver and healthcare providers regarding findings and care plans.

- Maintain the continuum of care by effectively coordinating the discharge with the primary care provider.

\section{SYSTEMS ORGANIZATION AND IMPROVEMENT}

In order to improve efficiency and quality within their organizations, pediatric hospitalists should:

- Coordinate the care of professional staff (including social work, nursing, speech or occupational therapists) and consultants to improve the quality and efficiency of care.

- Work with healthcare providers and community leaders to develop a system for effective and safe transitions of care from the inpatient to outpatient healthcare providers, preserving the multidisciplinary nature of the care team when appropriate.

- Lead, coordinate or participate in efforts to develop evidence-based guidelines for the evaluation and management of FTT in the hospital. 